(2)

(4)

(ㄷ)

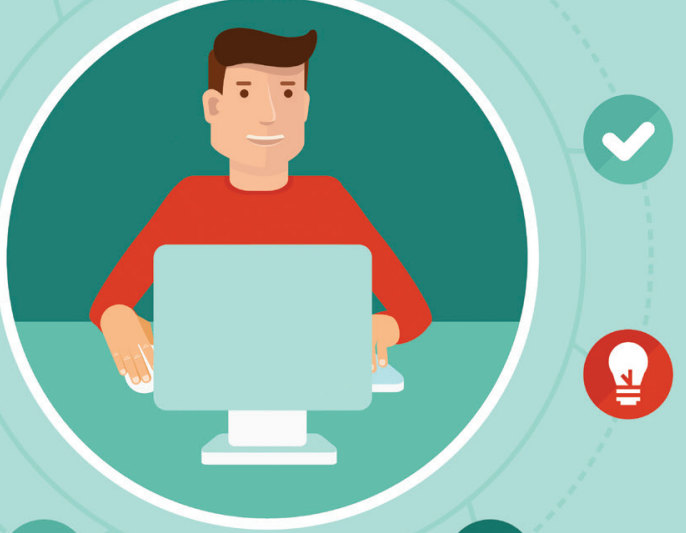

(i)
(ㅋ)

En los últimos cuatro años, la economía chilena ha mostrado la reciente crisis sub-prime de 2008. Llama la atención que en una desaceleración significativa en su ritmo de crecimiento, pa- los últimos cuatro años, sin haber entrado la economia chilena sando de crecer sobre 5\% en promedio en el periodo post crisis en una recesión, la proporción del empleo cuenta propia alcanzó sub-prime (2010-2013) a menos de 2\% en promedio entre 2014 niveles cercanos a los más altos observados después de la crisis y los meses que van de 2017. A pesar de ello, la tasa de desempleo ha aumentado sólo medio punto porcentual en los últimos cuatro años, siendo el registro más alto en este periodo de $7.1 \%$ y explicado en parte por factores estacionales. Este fenómeno llevo en su momento a plantear la idea que el mercado laboral estaba siendo resiliente frente a lo que pasaba con la actividad economica. Si se analiza la dinámica detrás de la tasa de desempleo se moderó también su crecimiento en los últimos años, empleos de manera suficiente como para empinar la tasa de desempleo a cifras mucho mayores que las observadas recientemente No obstante, el ajuste en el mercado laboral si se ha producido y lo ha hecho a través de cambios en la composición del empleo. En efecto, mientras el empleo asalariado creció menos de $1 \%$ en promedio desde 2014, el empleo por cuenta propia lo hizo a tasas superiores a 4\%. Con eso, la proporción del empleo total relacionado con actividades por cuenta propia se incrementó cerca de 4 puntos porcentuales en los uiltimos cuatro años (lo que representó un aumento de más de 370 mil trabajadores) y llegó a 22\% del empleo total. Estos cambios en la composición del empleo han hecho que la variabilidad de la tasa de desempleo tienda a estar contenida.

no es un fencisto expsicion del empleo cambie con el ciclo no es un fenomeno excepcional. En particular, la evidencia ha empleo por cuenta propia es contra-ciclico. Esto quiere decir que en expansiones económicas como la observada en la denominada época dorada de los commodities en los 2000, la incidencia del empleo asariado tiende a aumentar y la del empleo cuenta por propia se reduce. Lo contrario ocurre en recesiones. El empleo por cuenta propia como porcentaje del empleo total, se incremento sustantivamente en los dos últimos episodios de recesión vividos por la economia chilena. De acuerdo a la Figura 1, el empleo cuenta propia pasó de 18.4 a $22.3 \%$ del empleo total durante la crisis de fines de los noventas (crisis asiatica) y de 17.9 a 21.3\% durante

Ajustes recientes del mercado laboral:

\section{La tasa de} desempleo no, el empleo por cuenta propia sí.

Mauricio M. Tejada, académico FEN UAH ¿Qué significa ser trabajador por cuenta propia? Este tipo de empleo contiene un grupo altamente heterogéneo de trabajadoes. Por un lado, están los trabajadores con actividades laborales precarias y de muy baja remuneracion, mientras que por el otro

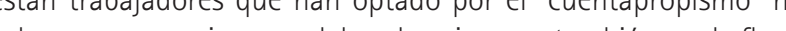
solo por mayores ingresos laborales, sino que tambien por la flex ta Según da tos de con bajos niveles de ingreso laboral a incidencia de empores cuenta propia es altamente significativa (ver Figura 2). En efecto más del 55 y el 23\% del empleo total en el primer y segundo decil de ingresos más bajos realiza actividades por cuenta propia respectivamente. Más aún, los trabajadores en estos dos deciles concentran mas del 35\% del total del empleo por cuenta propia. te ve constata nuevamente la importancia de este tipo de empleo para los trabajadores de baja calificación' ya que por cada 10 traajadores asalariados existen entre 5 y 6 trabajadores por cuenta propia (ver Higura 3). En el otro extremo, con menor escala, la inci-

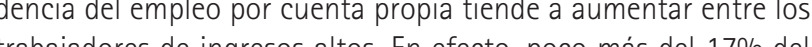
mpleo total es cuentas alopista en el decil de ingas del $17 \%$ del casi el 20\% del total del empleo por cuenta propia se encuentrá los ingresos más altos (ver Figura 2). Por nivel educativo, es notorio que el empleo asalariado es altamente relevante en el grupo de trabajadores calificados ya que por cada 10 trabajadores asalariados existen alrededor de 2 trabajadores por cuenta propia con educación superior y 1 con educación universitaria.

Las razones por las cuales la incidencia del empleo por cuenta propia es mas alta en estos dos grupos son diferentes. En el caso de los trabajadores menos educados y de bajos ingresos, el trabajo por cuenta propia representa la alternativa a un mercado labora cionalmente, este grupo no cuenta con ningún seguro de cesanti y por tanto el empleo por cuenta propia representa la forma de evitar el riesgo del desempleo. En el grupo de trabajadores más educados la historia es completamente diferente ya que si bien dos, el empleo cuenta propia representa una alternativa probablemente más rentable y que tiene beneficios adicionales como fexibilidad laboral y la autogestión del trabajo.

Otro patrón interesante está relacionado con la incidencia de "cuentapropismo" a lo largo del ciclo de vida de las personas (ver Figura 4). En efecto, la proporción de empleo cuenta propia aumenta con la edad. Dos interpretaciones pueden ser extraidas de esta observacion, de nuevo relacionadas con los diferentes tipos dores de bajos ingresos pareciera que las dificultades de optar por un empleo asalariado tienden a persistiry que este hecho se exacerba con la edad. En el grupo de trabajadores de altos ingresos, en tanto, la mayor incidencia con la edad puede estar relacionada además con un proceso de aprendizaje que tiende a hacer mas rentable la actividad por cuenta propia (respecto de la alternativa de conseguir un trabajo asalariado.

Con datos de la Encuesta de Protección Social 2015 (ver tabla 1), se observa que entre 2009 y 2016 el empleo por cuenta propia tendió a ser altamente persistente, ya que la probabilidad de mantenerse en este tipo de empleo en los siguientes seis meses fue 96\% y de poco más de $91 \%$ si se considera un ano. Más aún, probabilidad de obtener un empleo asalariado siendo cuentapropista fue de solo $1.4 \mathrm{y}$ 3\% a seis meses $y$ un año respectivamente. Finalmente, dejar el desempleo por un empleo por cuenta propi tiempo. Es destacable que estos numeros tienden a ser similares para diferentes niveles educativos del trabajidor.

En suma, si bien el empleo por cuenta propia es al tamente heterogéneo, la importancia que tiene el empleo de baja remuneración $y$ con trabajadores poco calificados hace que se le relacione con empleo precario e informal, y son justamente estos trabajadores vulnerables los que tienden a ser más afectados por los procesos de ajuste del mercado laboral en el ciclo económico. Más aún, si bien el empleo por cuenta propia representa una opción para sali del desempleo, este tiende a persistiry esto se exaceroa con ningún tipo de beneficios de la sequridad social.

' Trabajadodres sin educación o con solamente educación básica.

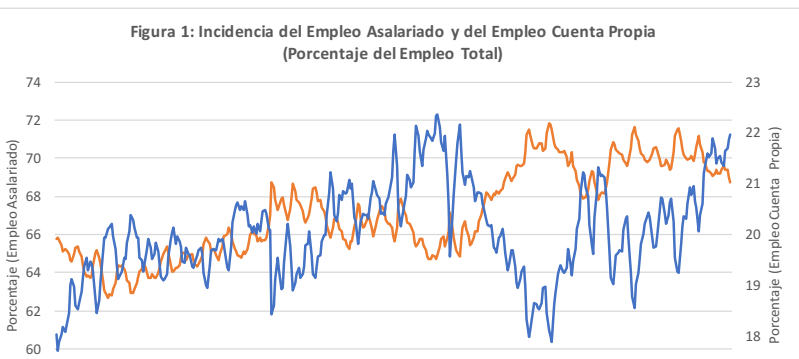

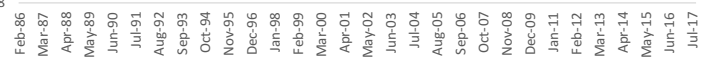

Figura a: : Empleo cuenta Propia por recil de Ingreso

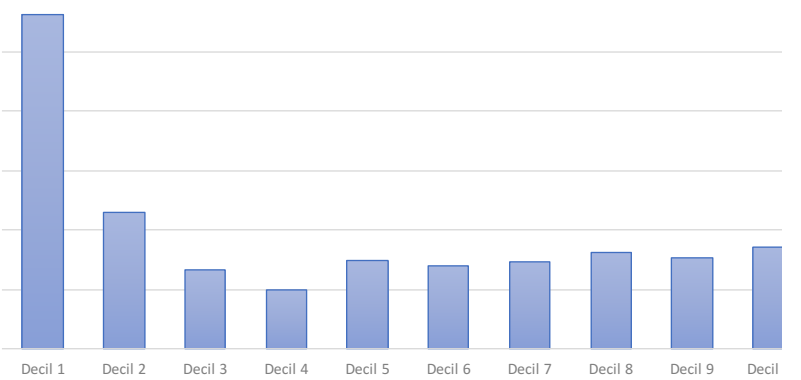

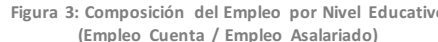

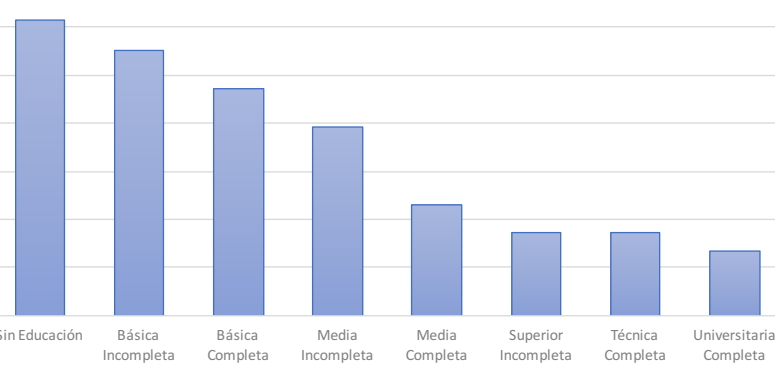

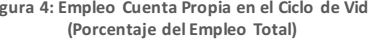

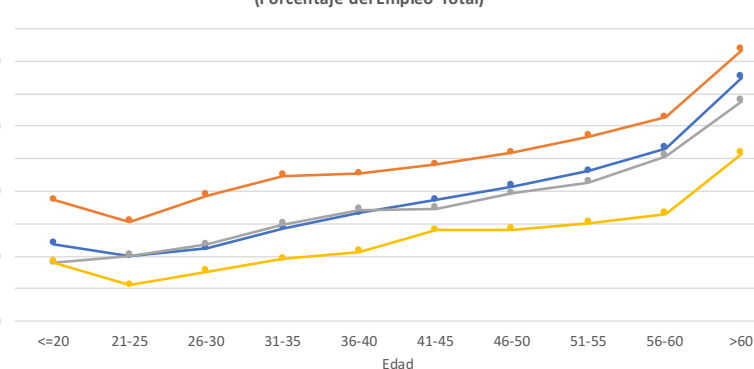

Probabilidades de Transición entre Estados del Mercado Laboral

Desempleado Cuenta Propia Asalariado Inactivo $\begin{array}{ll}\text { Asalariado } & 3.9 \\ \text { Inactivo } & 0.4\end{array}$

A un año

Desempleado $\quad 46.72 \quad 5.32$

$\begin{array}{lll}\text { Cuenta Propia } & 2.38 & 91.46 \\ \text { Asalariado } & 1.48 & 83.1\end{array}$

Inactivo

1.34

83.1
10.31 